\title{
Spontaneous crystallization of skyrmions and fractional vortices in fast-rotating and rapidly quenched spin-1 Bose-Einstein condensates
}

\author{
S.-W. Su, ${ }^{1}$ C.-H. Hsueh, ${ }^{2}$ I.-K. Liu, ${ }^{3}$ T.-L. Horng, ${ }^{4}$ Y.-C. Tsai, ${ }^{5}$ S.-C. Gou, ${ }^{3}$ and W. M. Liu ${ }^{6}$ \\ ${ }^{1}$ Department of Physics, National Tsing Hua University, Hsinchu 30013, Taiwan \\ ${ }^{2}$ Department of Physics, National Taiwan Normal University, Taipei 11677, Taiwan \\ ${ }^{3}$ Department of Physics, National Changhua University of Education, Changhua 50058, Taiwan \\ ${ }^{4}$ Department of Applied Mathematics, Feng Chia University, Taichung 40724, Taiwan \\ ${ }^{5}$ Department of Photonics, Feng Chia University, Taichung 40724, Taiwan \\ ${ }^{6}$ Instuite of Physics, Chinese Science Academy, Beijing 100190, People's Republic of China
}

(Received 22 May 2011; published 1 August 2011)

\begin{abstract}
We investigate the spontaneous generation of crystallized topological defects via the combining effects of fast rotation and rapid thermal quench on spin-1 Bose-Einstein condensates (BECs). By solving the stochastic projected Gross-Pitaevskii equation, we show that, when the system reaches equilibrium, a hexagonal lattice of skyrmions and a square lattice of half-quantized vortices can be formed in a ferromagnetic and antiferromagnetic spinor BEC, respectively, which can be imaged by using the polarization-dependent phase-contrast method.
\end{abstract}

\section{INTRODUCTION}

Topological defects are a manifestation of spontaneously broken symmetries [1]. The formation and observation of topological defects is one of the most fundamental and fascinating topics in various aspects of physics, ranging from condensed-matter physics to cosmology. However, owing to the limitation of energy scales in earth-bound physics experiments, topological defects are mostly created and observed in condensed-matter systems. For example, magnetic domains, walls of magnetized material, and string defects in ${ }^{3} \mathrm{He}$ superfluid phase transitions have been extensively studied [2].

Recently, owing to the realization of spinor Bose-Einstein condensates (BECs) of alkali-metal atoms in an optical trap $[3,4]$, the creation of topological defects in ultracold atomic systems has become possible. A spinor BEC is fully characterized by the spin degrees of freedom and behaves as a vector in the spin space. Theoretical studies for the spinor BEC were pioneered by Ho [5] and independently by Ohmi and Machida [6]. More recently, spinor BECs with $F>1$ [7-9] or with long-range dipolar interaction [10,11] have also been theoretically investigated. An overview on the physics of spinor BEC systems can be found in Ref. [12]. In general, the physical behavior of the spinor BEC depends crucially on its magnetic properties, such that the interplay between the superfluidity and the magnetism of the condensate makes the spinor BEC a candidate to exhibit a variety of nontrivial ordered states, such as skyrmions [13], Mermin-Ho vortices [14], Anderson-Thouless vortices [15], and so forth.

So far, all theoretical studies regarding the formation of topological defects in spinor BECs appear to manipulate the external and internal degrees of freedom of the condensed atoms at zero temperature. On the other hand, according to the Kibble-Zurek scenario, topological defects can also be created through phase transitions at finite temperatures, which are fundamentally caused by spontaneous symmetry breaking and thermal fluctuations near the critical point. In this paper, we show that it is possible to create crystalline orders of skyrmions and fractional vortices simply by thermally quenching a rotating spin-1 BEC. This enables us to probe into the very fundamental aspects of topological defects without any engineering of dynamical processes, since evaporative cooling is a prerequisite in creating BECs and the methods of rotating condensates have been well developed in a variety of ultracold atomic experiments. In the framework of mean-field theory, the dynamics of a BEC at nonzero temperatures can be described by the stochastic projected Gross-Pitaevskii equation (SPGPE) [16], which relies on the assumption that the system can be treated as a condensate band in contact with a thermal reservoir comprising all noncondensed particles. In such a scheme, the condensate band is described by the truncated Wigner method [17] including the projected $c$-field method, while the noncondensate band is described by quantum kinetic theory $[18,19]$. In the following, we solve the SPGPE numerically for a rotating trapped spin-1 BEC. We show that when the system is quenched down to a very low temperature a lattice of skyrmions and half-quantized vortices (HQVs) can be created in the spinor BEC of ${ }^{87} \mathrm{Rb}$ and ${ }^{23} \mathrm{Na}$, respectively.

\section{FORMALISM}

The spin-1 BEC is characterized by a vectorial order parameter, $\Psi=\left(\Psi_{1}, \Psi_{0}, \Psi_{-1}\right)^{T}$ (the superscript $T$ stands for the transpose), where the components $\Psi_{j}(j= \pm 1,0)$ denotes the macroscopic wave function of the atoms condensed in the spin state, $\left|F=1, m_{F}=j\right\rangle \equiv|1, j\rangle$, that can be equivalently expressed as $\Psi_{j}=|\Psi(\mathbf{r})| \varsigma_{j}(\mathbf{r})$, with $\varsigma_{j}(\mathbf{r})$ being a threecomponent spinor, normalized to the condition $\sum_{j} \varsigma_{j}^{\dagger} \varsigma_{j}=1$. The dynamics of $\Psi_{j}$ in a confining potential is described by the following coupled nonlinear Schrödinger equations:

$$
\begin{aligned}
i \hbar \partial_{t} \Psi_{j} & =\hat{H}_{j}^{\mathrm{GP}} \Psi_{j} \\
& =\hat{\mathcal{H}} \Psi_{j}+g_{s} \sum_{\alpha=x, y, z} \sum_{n, k, l=0, \pm 1}\left(\hat{F}_{\alpha}\right)_{j n}\left(\hat{F}_{\alpha}\right)_{k l} \Psi_{n} \Psi_{k}^{*} \Psi_{l},
\end{aligned}
$$

where $\hat{\mathcal{H}}=-\hbar^{2} \nabla^{2} / 2 m+V(\mathbf{r})+g_{n}|\Psi|^{2}$ denotes the spinindependent part of the Hamiltonian, and $\hat{F}_{\alpha}$ are the matrices 
representing the Cartesian components of the spin angular momentum with quantization axis fixed in the $z$ axis, namely,

$$
\begin{gathered}
\hat{F}_{x}=\frac{1}{\sqrt{2}}\left(\begin{array}{ccc}
0 & 1 & 0 \\
1 & 0 & 1 \\
0 & 1 & 0
\end{array}\right), \quad \hat{F}_{y}=\frac{1}{\sqrt{2}}\left(\begin{array}{ccc}
0 & -i & 0 \\
i & 0 & -i \\
0 & i & 0
\end{array}\right), \\
\hat{F}_{z}=\left(\begin{array}{ccc}
1 & 0 & 0 \\
0 & 0 & 0 \\
0 & 0 & -1
\end{array}\right) .
\end{gathered}
$$

The coupling constants $g_{n}$ and $g_{s}$ characterizing the densitydensity and spin-exchange interactions, respectively, are related to the $s$-wave scattering lengths $a_{0}$ and $a_{2}$ in the total spin channels $F_{\text {tot }}=0$ and $F_{\text {tot }}=2$ by $g_{n}=4 \pi \hbar^{2}\left(a_{0}+2 a_{2}\right) / 3 m$, $g_{s}=4 \pi \hbar^{2}\left(a_{2}-a_{0}\right) / 3 m[5,6]$. The ground state of the spinor BEC depends crucially on the sign of $g_{s}$. For the ferromagnetic coupling, $g_{s}<0$, the condensate is in the "axial" state, $|\langle\hat{\mathbf{F}}\rangle|=1$, with $\langle\hat{\mathbf{F}}\rangle=\sum_{i j} \varsigma_{i}^{\dagger}(\hat{\mathbf{F}})_{i j} \varsigma_{j}$ [5]. On the other hand, for the antiferromagnetic coupling, $g_{s}>0$, the condensate is in the "polar" state, $\langle\hat{\mathbf{F}}\rangle=0$ [5]. Because we focus on the vortices formed by the spin textures, it is more convenient to introduce the basis kets $|\alpha\rangle(\alpha=x, y, z)$ which satisfy the conditions $\hat{F}_{\alpha}|\alpha\rangle=0$. It is straightforward to show that $|z\rangle=|1,0\rangle$, and

$$
\begin{gathered}
|x\rangle=\exp \left(-i \frac{\pi}{2} \hat{F}_{y}\right)|z\rangle=\frac{1}{\sqrt{2}}(-|1,1\rangle+|1,-1\rangle), \\
|y\rangle=\exp \left(i \frac{\pi}{2} \hat{F}_{y}\right)|z\rangle=\frac{i}{\sqrt{2}}(|1,1\rangle+|1,-1\rangle)
\end{gathered}
$$

Accordingly, we have $\Psi_{ \pm 1}=\left( \pm \Psi_{x}+i \Psi_{y}\right) / \sqrt{2}$ and $\Psi_{0}=$ $\Psi_{z}$. The spin texture, which is parallel to the local magnetic moment, is defined by $\mathbf{S}(\mathbf{r})=i \rho^{-1} \tilde{\boldsymbol{\Psi}}^{\dagger} \times \tilde{\boldsymbol{\Psi}}$, where $\tilde{\boldsymbol{\Psi}}=\left(\Psi_{x}, \Psi_{y}, \Psi_{z}\right)^{T}$ [20]. Consequently, we have $S_{x} \propto$ $\operatorname{Re}\left[\left(\Psi_{1}+\Psi_{-1}\right) \Psi_{0}^{*}\right], \quad S_{y} \propto \operatorname{Re}\left[\mathrm{i}\left(\Psi_{1}-\Psi_{-1}\right) \Psi_{0}^{*}\right], \quad$ and $S_{z} \propto$ $\left|\Psi_{1}\right|^{2}-\left|\Psi_{-1}\right|^{2}$. For later convenience, we define the unit vector $\mathbf{s}(\mathbf{r})=\mathbf{S}(\mathbf{r}) /|\mathbf{S}(\mathbf{r})|$.

To study the nonequilibrium dynamics of a quenched rotating spinor BEC, we generalize the formulas in Ref. [16] to the following set of coupled SPGPEs:

$$
\partial_{t} \Psi_{j}=\mathcal{P}\left\{-\frac{i}{\hbar} \hat{H}_{j}^{\mathrm{GP}} \Psi_{j}+\frac{\gamma_{j}}{k_{B} T}\left(\mu-\hat{H}_{j}^{\mathrm{GP}}\right) \Psi_{j}+\frac{d W_{j}}{d t}\right\},
$$

where $T$ and $\mu$ denote the final temperature and chemical potential, $\gamma_{j}$ the growth rate for the $j$ th component, and $d W_{j} / d t$ is the complex-valued white noise associated with the condensate growth. The projection operator $\mathcal{P}$ restricts the dynamics of the spinor BEC in the lower-energy region below the cutoff energy $E_{R}$. In the rotating frame, $\hat{\mathcal{H}}$ is replaced by $\hat{\mathcal{H}}-\Omega \hat{L}_{z}$, where $\hat{L}_{z}=-i \hbar\left(x \partial_{y}-y \partial_{x}\right)$ is the $z$ component of the orbital angular momentum, and $\Omega$ is the angular frequency of rotation. Because we focus on the fast-rotating BECs, in which the atomic cloud is shaped like a two-dimensional (2D) droplet, it is reasonable to treat the system as two-dimensional. We therefore assume $V(\mathbf{r})=m \omega^{2}\left(x^{2}+y^{2}+\lambda^{2} z^{2}\right) / 2$ with $\lambda \gg 1$. The effective 2D interaction strength can be obtained by integrating the wave functions with respect to $z$ to eliminate the axial degree of freedom. The numerical procedures for integrating the set of coupled SPGPEs are described as follows. First, the initial state of each $\Psi_{j}$ is sampled by using the grandcanonical ensemble for a free ideal Bose gas at a temperature $T_{0}$ below the critical temperature and of chemical potentials $\mu_{j, 0}$. The spatial dependence of the initial state can be specified as a linear combination of some basis functions. Here, we adopt the basis consisting of plane waves with quantized momentum $\mathbf{k}=2 \pi\left(n_{x}, n_{y}\right) / L$ (where $n_{x}, n_{y}$ are integers and $L$ is the size of the computation domain); i.e., $\Psi_{j}(t=0)=\sum_{\mathbf{k}}^{E_{R}} a_{j ; \mathbf{k}} \psi_{\mathbf{k}}(\mathbf{r})$, where $\psi_{\mathbf{k}}(\mathbf{r})$ are the plane-wave basis functions. The condensate band lies below an energy cutoff $E_{R}>E_{\mathbf{k}}=\hbar^{2}|\mathbf{k}|^{2} / 2 m$. Furthermore, the distribution is sampled by $a_{j, \mathbf{k}}=\left(N_{j, \mathbf{k}}+\right.$ $1 / 2)^{1 / 2} \eta_{j, \mathbf{k}}$, where $N_{j, \mathbf{k}}=\left\{\exp \left[\left(E_{j, \mathbf{k}}-\mu_{j .0}\right) / k_{B} T_{0}\right]-1\right\}^{-1}$ and $\eta_{j, \mathbf{k}}$ are the complex Gaussian random variables with moments $\left\langle\eta_{j, \mathbf{k}} \eta_{j, \mathbf{k}^{\prime}}\right\rangle=\left\langle\eta_{j, \mathbf{k}}^{*} \eta_{j, \mathbf{k}^{\prime}}^{*}\right\rangle=0$ and $\left\langle\eta_{j, \mathbf{k}} \eta_{j, \mathbf{k}^{\prime}}^{*}\right\rangle=\delta_{\mathbf{k k}^{\prime}}$. Second, to simulate the thermal quench, the temperature and chemical potential of the noncondensate band are altered to the new values $T<T_{0}$ and $\mu>\mu_{j, 0}$. For convenience, we adopt the oscillator units in the numerical computations, where the length, time, and energy are respectively scaled in units of $\sqrt{\hbar / m \omega}, \omega^{-1}$, and $\hbar \omega$.

\section{RESULTS AND DISCUSSIONS}

We first study the spinor BEC of ${ }^{87} \mathrm{Rb}$, which has $g_{s}<0$. The total number of the modes are $n_{x}, n_{y}=256$ and the energy cutoff is chosen at $n_{x c}, n_{y c}=128$. The parameters are $\Omega=0.95, T=10 \mathrm{nK}, \mu=8$, and $\hbar \gamma_{j} / k_{B} T=0.03$ for all $\Psi_{j}$ 's. The time evolutions of the density profiles for $\Psi_{j}$ 's are shown in Fig. 1. During the evaporative cooling, the rotating condensates grow up and the emergent vortices start closely binding up and forming vortex trimers in each $\Psi_{j}$. When the system reaches equilibrium, these vortex trimers arrange themselves into some interwoven lattice structures such that each vortex core of $\Psi_{j}$ is filled up with particles of the remaining two components. In other words, quantized vortices,
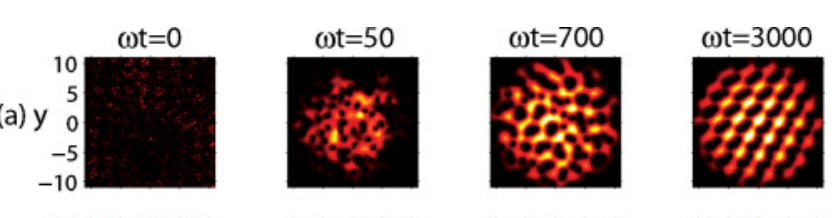

(b)
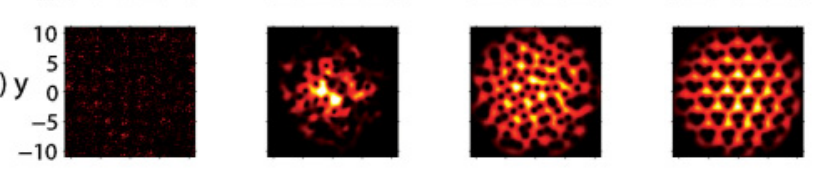

(c) $y$
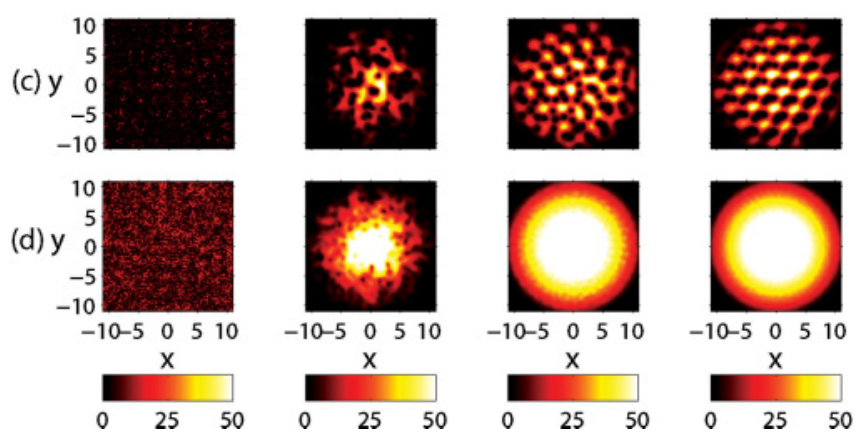

FIG. 1. (Color online) Snapshots of (a) $\left|\Psi_{-1}\right|^{2}$, (b) $\left|\Psi_{0}\right|^{2}$, (c) $\left|\Psi_{1}\right|^{2}$, and (d) $|\Psi|^{2}$ for the quenched rotating spinor BEC of ${ }^{87} \mathrm{Rb}$. When the system reaches equilibrium at $T=10 \mathrm{nK}$ with $\Omega=0.95$, $\mu=8$ (rightmost column), the crystalline order of vortex trimers is established in each $\Psi_{j}$. The particle numbers in the spinor BEC are $N_{ \pm 1} \approx 4.84 \times 10^{3}, N_{0} \approx 4.79 \times 10^{3}$. 

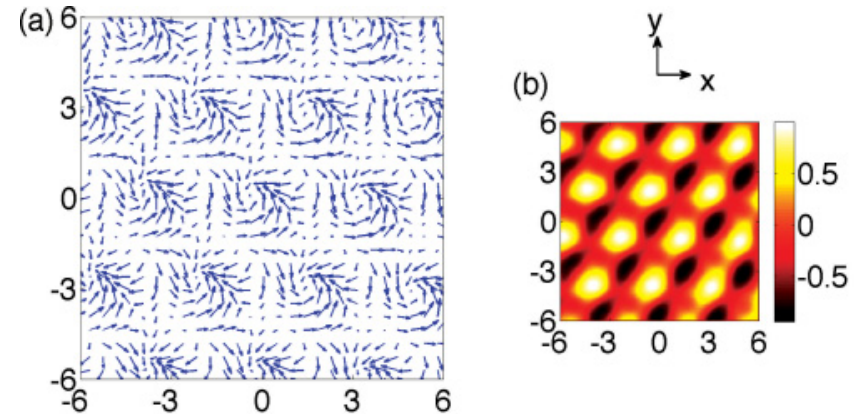

(c)
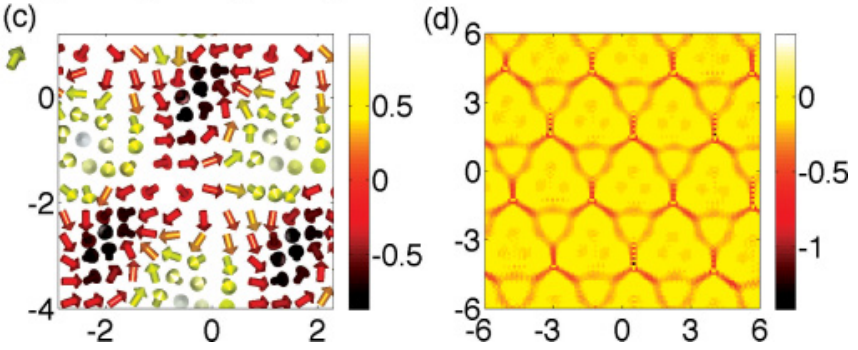

FIG. 2. (Color online) (a) The equilibrium spin textures for the spinor BEC of ${ }^{87} \mathrm{Rb}$. (b) Distribution of $S_{z}$ in the spin textures. (c) The orientations of the unit vector $\mathbf{s}(\mathbf{r})$ for a skyrmion. The color of each arrow indicates the magnitude of $S_{z}$. (d) The density profile of the topological charges.

either inter- or intraspecies, avoid overlapping with each other. The shapes of the trimer structures in all components are somewhat different. To characterize these structures, we calculate the incompressible kinetic energy per particle for each $\Psi_{j}$. This can be done by writing $\Psi_{j}=\left|\Psi_{j}\right| \exp \left(i \varphi_{j}\right)$ and defining the current $\mathbf{Z}_{j}=\left|\Psi_{j}\right| \nabla \varphi_{j}=\mathbf{Z}_{j}^{(i)}+\mathbf{Z}_{j}^{(c)}[21]$ in terms of the solenoidal and irrotational fields, where $\nabla \cdot \mathbf{Z}_{j}^{(i)}=$ $0, \nabla \times \mathbf{Z}_{j}^{(c)}=\mathbf{0}$. The incompressible energy is defined by $\mathcal{E}_{k, j}^{(i)}=(1 / 2) \int d^{2} r\left|\mathbf{Z}_{j}^{(i)}\right|^{2}$, which corresponds to the kinetic energy of swirls in the superflows. Consequently, we find that $\mathcal{E}_{k, j}^{(i)} / N_{j}=19.48,19.27,20.05$ for $j= \pm 1,0$, respectively. The spin textures are shown in Fig. 2(a), where a hexagonal lattice is visualized. Furthermore, the spatial variation of the local spin moments parallel to the axis of rotation is plotted in Fig. 2(b). An enlarged perspective view of the three-dimensional orientations of the local spins is shown in Fig. 2(c). Taking the island around the trap center, for example, we see that the innermost spin points into the paper, while the others increasingly twist and bend in the upward direction. This vortexlike arrangement of magnetic moments is exactly the configuration of a skyrmion [22-24]. Since the central spin in the skyrmion is perpendicular to the rotating plane, $S_{x}$ and $S_{y}$ must vanish therein, implying that the skyrmions must be centered at the regions of $\Psi_{0}=0$, i.e., the cores of the vortex trimer in $\Psi_{0}$. The topological charge density, $\sigma=\mathbf{s} \cdot(\partial \mathbf{s} / \partial x \times \partial \mathbf{s} / \partial y) / 4 \pi$ [23], is shown in Fig. 2(d), which exhibits a hexagonal lattice structure. Numerical integration over the primitive unit cell reveals that each skyrmion carries a topological charge $Q=-1$. We notice that the crystallization of skyrmions has recently been observed in various magnetic materials characterized by the Dzyaloshinskii-Moriya interaction [23,24]. Amazingly, the
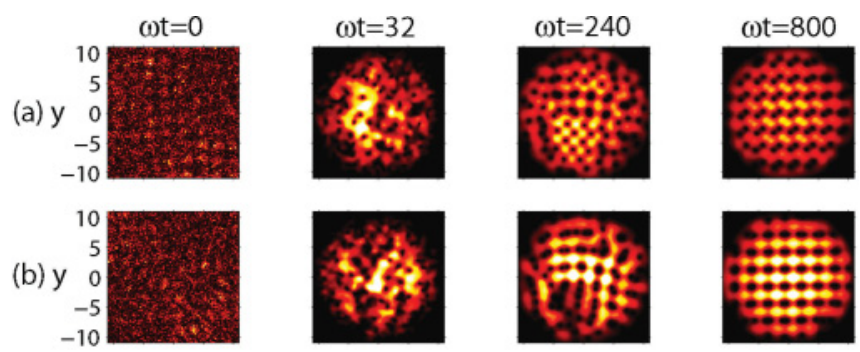

(c) $\mathrm{y}$
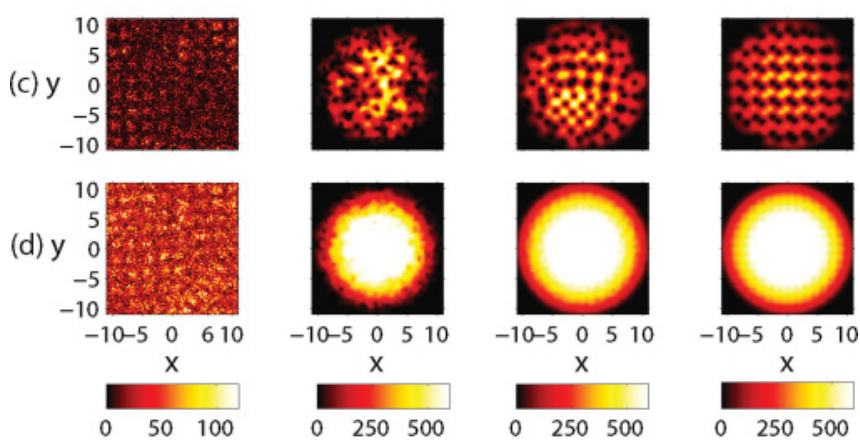

FIG. 3. (Color online) Snapshots of (a) $\left|\Psi_{-1}\right|^{2}$, (b) $\left|\Psi_{0}\right|^{2}$, (c) $\left|\Psi_{1}\right|^{2}$, and (d) $|\Psi|^{2}$ for a quenched rotating spinor BEC of ${ }^{23} \mathrm{Na}$. When the system reaches equilibrium at $T=10 \mathrm{nK}$ with $\Omega=0.8$, $\mu=25$ (rightmost column), the crystalline order of vortex dimers is established in each $\Psi_{j}$. The particle numbers in the spinor BEC are $N_{ \pm 1} \approx 4.55 \times 10^{4}, N_{0} \approx 7.70 \times 10^{4}$.

spin textures in these magnetic materials highly resemble those in Fig. 2(a).

For the case of $g_{s}>0$, we consider the spinor BECs of ${ }^{23} \mathrm{Na}$. We set $\Omega=0.8, T=10 \mathrm{nK}, \mu=25$, and $\hbar \gamma_{j} / k_{B} T=0.03$ for all spin components. The total number of modes and energy cutoff remain the same as those for the case of ${ }^{87} \mathrm{Rb}$. In Fig. 3, the time evolutions of the density profiles for $\Psi_{j}$ are shown. The nucleation of the vortices in the current case is similar to that in the case of ${ }^{87} \mathrm{Rb}$, except that a square lattice of tightly bound vortex dimers is formed in each component. Consequently, we find that $\mathcal{E}_{k, j}^{(i)} / N_{j}=13.44,13.45,12.54$ for $j= \pm 1,0$, respectively. The equilibrated spin textures on the rotating plane are shown in Fig. 4(a), in which a mosaic of magnetic domains with staggered magnetization is created. In Figs. 4(b) and 4(c), we see that almost all spins in the magnetic domains lie in the $x y$ plane. Spins belonging to the same domain align nearly unidirectionally. Note that the spins reverse their magnetization through a Bloch wall transition in a very narrow region near the boundary between two adjacent domains. These staggered magnetic domains act as the smoking gun of HQVs, which have been predicted to exist in superfluid ${ }^{3} \mathrm{He}$ [2] and superconductors [25]. Considering the transformation $\boldsymbol{\Psi} \rightarrow \hat{G}(\theta) \hat{R}(\mathbf{n}, \chi) \boldsymbol{\Psi}$, where $\hat{G}(\theta)=\exp (i \theta)$ is a gauge transformation and $\hat{R}(\mathbf{n}, \chi)=\exp (i \chi \mathbf{n} \cdot \hat{\mathbf{F}})$ is a spin rotation through an angle $\chi$ about the unit vector $\mathbf{n}$, a HQV entails a spin rotation with $\chi=\pi$ followed by a global phase change of $\pi$ in $\boldsymbol{\Psi}$. Without loss of generality, we assume $s(\mathbf{r})=\cos \phi(\mathbf{r}) \mathbf{e}_{x}+\sin \phi(\mathbf{r}) \mathbf{e}_{y}$. Numerically, we verify that the spin textures remain unchanged through the transformation $\hat{G}(\pi) \hat{R}(\mathbf{s}, \pi) \Psi=\left(e^{-2 i \phi(\mathbf{r})} \Psi_{-1}, \Psi_{0}, e^{2 i \phi(\mathbf{r})} \Psi_{1}\right)^{T}$. Upon requiring $\hat{G}(\pi) \hat{R}(\mathbf{s}, \pi) \boldsymbol{\Psi}=e^{i 2 \pi} \boldsymbol{\Psi}$, it follows that $e^{-2 i \phi(\mathbf{r})} \Psi_{-1}=\Psi_{1}$ 

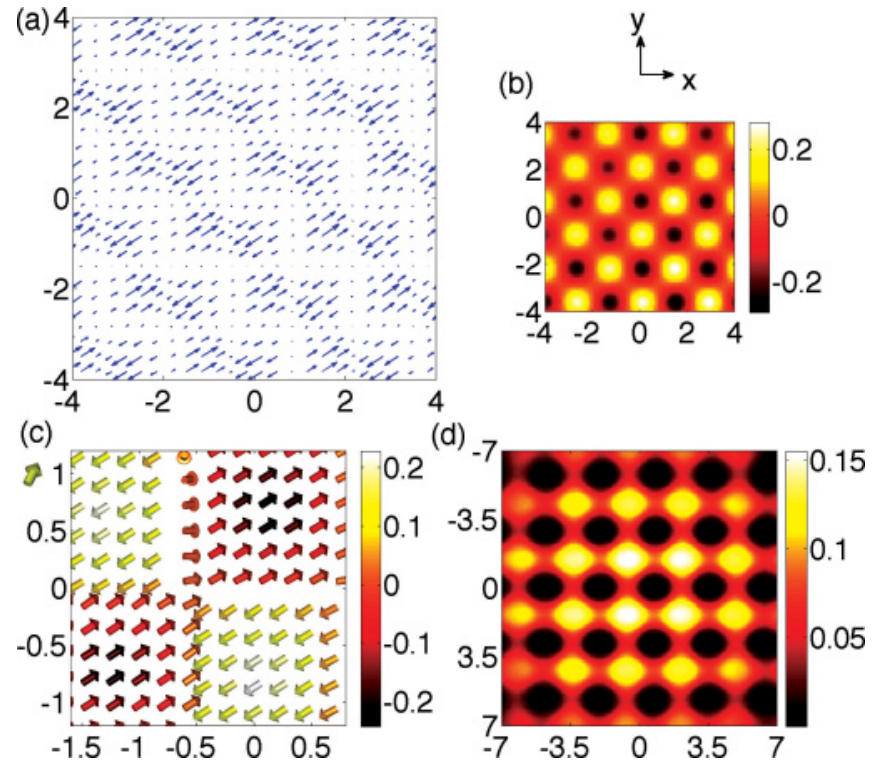

FIG. 4. (Color online) (a) The equilibrium spin textures for the spinor BEC of ${ }^{23} \mathrm{Na}$. (b) Distribution of $S_{z}$ in the spin textures. (c) The orientations of the unit vector $\mathbf{s}(\mathbf{r})$ in the adjacent magnetic domains. The color of each arrow indicates the magnitude of $S_{z}$. (d) The modulus of $\left|\Psi_{1}-e^{-2 i \phi(\mathbf{r})} \Psi_{-1}\right|$, where dark shaded regions reveal the locations of HQVs.

and $e^{2 i \phi(\mathbf{r})} \Psi_{1}=\Psi_{-1}$. The redundancy of the last equality implies that there are multiple solutions satisfying the criterion, $\left|\Psi_{1}-e^{-2 i \phi(\mathbf{r})} \Psi_{-1}\right|=0$, or equivalently, $\left|\Psi_{-1}\right|=\left|\Psi_{1}\right|$. In Fig. 4(d), the modulus $\left|\Psi_{1}-e^{-2 i \phi(\mathbf{r})} \Psi_{-1}\right|$ is plotted where the dark shaded areas represent the core positions of HQVs, which form a square lattice apparently. Likewise, the HQVs are localized at the cores of vortex dimers in $\Psi_{0}$. Our results are consistent with those obtained by means of dynamical creation [26], where a lattice of HQVs can be created in a rotating optical trap when additional pulsed magnetic trapping potentials are applied. Furthermore, the ground state of a rotating dipolar spinor BEC has been shown to have the same structure when the dipole-dipole interaction is small compared to the contact ones [27].
We note that an $\Omega$ comparable to $\omega$ is needed to stabilize the crystalline orders of defects. Under such a fast rotation, the filling factor $v$, i.e., the ratio of the number of atoms to the number of vortices, can have a value of a few hundreds for each component, as shown in Figs. 1 and 3. According to the criterion in Ref. [28], the system enters the mean-field quantum Hall regime, in which the mean-field theory still applies yet the state of the system can be well described in the lowest Landau-level approximation. When $\Omega$ is not sufficiently large, the crystallization does not arise, albeit some few topological defects may be readily created in the condensate. For example, in our simulations with $\Omega=0.3$, we find only a few HQVs nucleating in the spinor $\mathrm{BEC}$ of ${ }^{23} \mathrm{Na}$ during the rapid rotational evaporative cooling. The situation is somewhat different in the case of ${ }^{87} \mathrm{Rb}$, where Mermin-Ho vortices, rather than the skyrmions, are created in the condensate. Furthermore, to see whether the crystallization is robust against the thermal fluctuations arising from the growth of condensate, we have assumed a range of final equilibrium temperatures in the simulations. We find that both crystalline orders remain intact for temperatures lower than $50 \mathrm{nK}$. At higher temperatures, however, the crystallization is thwarted by the fluctuations of spin textures so the lattice becomes disordered and starts melting when the system approaches the critical regime.

\section{CONCLUSIONS}

In summary, we have investigated the nonequilibrium dynamics of spin-1 BECs during rapid rotational evaporative cooling. Crystallization of skyrmions and HQVs is predicted to arise in the spinor BEC of ${ }^{87} \mathrm{Rb}$ and ${ }^{23} \mathrm{Na}$, respectively. To resolve the spatial magnetization of the crystallized topological defects, the images have to be taken in situ, and this can be achieved basically by using the polarization-dependent phase-contrast technique [29].

\section{ACKNOWLEDGMENTS}

S.-C. Gou is supported by NSC Taiwan, under Grant No. 98-2112-M-018-001-MY2, and partially by NCTS. W. M. Liu is supported by NSFC under Grant No. 10934010, and by NKBRSFC under Grant No. 2011CB921502.
[1] G. E. Volovik, Exotic Properties of Superfluid ${ }^{3} \mathrm{He}$ (World Scientific, Singapore, 1992).

[2] G. E. Volovik, The Universe in a Helium Droplet (Clarendon, Oxford, 2003).

[3] D. M. Stamper-Kurn, M. R. Andrews, A. P. Chikkatur, S. Inouye, H. J. Miesner, J. Stenger, and W. Ketterle, Phys. Rev. Lett. 80, 2027 (1998).

[4] M. D. Barrett, J. Sauer, and M. S. Chapman, Phys. Rev. Lett. 87, 010404 (2001).

[5] T.-L. Ho, Phys. Rev. Lett. 81, 742 (1998).

[6] T. Ohmi and K. Machida, J. Phys. Soc. Jpn. 67, 1822 (1998).

[7] M. Ueda and M. Koashi, Phys. Rev. A 65, 063602 (2002).

[8] H. Schmaljohann, M. Erhard, J. Kronjager, M. Kottke, S. van Staa, L. Cacciapuoti, J. J. Arlt, K. Bongs, and K. Sengstock, Phys. Rev. Lett. 92, 040402 (2004).
[9] H. Saito and M. Ueda, Phys. Rev. A 72, 053628 (2005).

[10] M. Yasunaga and M. Tsubota, Phys. Rev. Lett. 101, 220401 (2008)

[11] S. Hoshi and H. Saito, Phys. Rev. A 81, 013627 (2010).

[12] M. Ueda and Y. Kawaguchi, e-print arXiv:1001.2072.

[13] U. Al Khawaja and H. T. C. Stoof, Phys. Rev. A 64, 043612 (2001).

[14] N. D. Mermin et al., Phys. Rev. Lett. 36, 594 (1976).

[15] P. W. Anderson et al., Phys. Rev. Lett. 38, 508 (1977).

[16] A. S. Bradley, C. W. Gardiner, and M. J. Davis, Phys. Rev. A 77, 033616 (2008).

[17] A. Sinatra et al., J. Phys. B 35, 3599 (2002).

[18] C. W. Gardiner and P. Zoller, Phys. Rev. A 58, 536 (1998).

[19] C. W. Gardiner and P. Zoller, Phys. Rev. A 61, 033601 (2000). 
[20] T. Mizushima, N. Kobayashi, and K. Machida, Phys. Rev. A 70, 043613 (2004).

[21] T.-L. Horng, C.-H. Hsueh, S.-W. Su, Y.-M. Kao, and S.-C. Gou, Phys. Rev. A 80, 023618 (2009).

[22] U. K. Röbler et al., Nature (London) 442, 797 (2006).

[23] S. Mühlbauer et al., Science 323, 915 (2009).

[24] X. Z. Yu et al., Nature (London) 465, 901 (2010).

[25] D. A. Ivanov, Phys. Rev. Lett. 86, 268 (2001).
[26] A.-C. Ji, W. M. Liu, J. L. Song, and F. Zhou, Phys. Rev. Lett. 101, 010402 (2008).

[27] T. P. Simula et al., J. Phys. Soc. Jpn. 80, 013001 (2011).

[28] T.-L. Ho, Phys. Rev. Lett. 87, 060403 (2001).

[29] J. M. Higbie, L. E. Sadler, S. Inouye, A. P. Chikkatur, S. R. Leslie, K. L. Moore, V. Savalli, and D. M. Stamper-Kurn, Phys. Rev. Lett. 95, 050401 (2005). 